\title{
New Insights into the Three-Dimensional Anatomy of the Facial Mimetic Muscles Related to the Nasolabial Fold: An Iodine Staining Technique Based on Nano-computed Tomography (Discussion)
}

\author{
Sergey Y. Turin ${ }^{1} \cdot$ Arun K. Gosain $^{1}$ (1)
}

Received: 18 November 2019/Accepted: 18 November 2019/Published online: 10 December 2019

(C) Springer Science+Business Media, LLC, part of Springer Nature and International Society of Aesthetic Plastic Surgery 2019

Level of Evidence $V$ This journal requires that authors assign a level of evidence to each article. For a full description of these Evidence-Based Medicine ratings, please refer to the Table of Contents or the online Instructions to Authors www.springer.com/00266.

The nasolabial fold (NLF) is a key landmark for facial animation and rejuvenation. A number of investigations into the anatomy of the nasolabial fold have been carried in the preceding decades [1-3], and this continues to be an area of active research for multiple specialties [4] and applications $[5,6]$.

The investigators have carried out an anatomic study of the soft tissues in the NLF region, with a specific focus on the facial mimetic muscles (FMMs) thought to be responsible for NLF formation. The authors used nano-CT technology to study the relationships of the FMMs in situ, which is an interesting evolution of using cross-sectional imaging to examine these delicate structures.

The authors do a laudable job of describing the limitations of the study, which are mostly due to the specimen size constraints imposed by the nano-CT technology. In the effort to obtain the smallest possible specimen to optimize the resolution of the scans, the specimens examined were harvested from three fetuses of 20-25 weeks gestational age and consisted of hemi-facial soft tissues elevated off the bony skeleton. These are notable considerations for interpreting the results: It is difficult to correlate the size and shape of the FMMs in fetal specimens to adults, or to

Arun K. Gosain

argosain@luriechildrens.org

1 Division of Pediatric Plastic Surgery, Lurie Childrens Hospital of the Northwestern University Feinberg School of Medicine, 225 E. Chicago Ave., Box 93, Chicago, IL, USA account for the changes that occur as a result of removing the underlying skeletal scaffold. Moreover, some of the fascial planes the authors describe may change with time. However, it seems reasonable to assume the overall architecture of the FMM insertions is likely maintained, as we are not aware of any evidence to show that the relationships of muscles to each other and their underlying structures change once established.

In their results, the authors give some insight into the fascial relationship in the NLF area by noting that the "Adipose tissue in the cheek was not a mass of unorganized fat. It was separated and fixed by branch fibers." We have previously noted the importance of the distinct adipofascial layer in this area [2] and believe the authors are characterizing a subsection of this layer. As we have proposed, it can act to effectively transmit a vector of pull to the NLF and smooth it out in facial rejuvenation scenarios. The authors' current description of this relationship in situ may be the next step in developing more efficacious methods of exerting force on the soft tissues around the NLF in a targeted fashion.

The authors' findings on FMM anatomy are summarized in Fig. 3, where they show that instead of a confluence of muscles at the modiolus, there is an organized sequence of these muscles inserting on each other: The zygomaticus major inserts onto the levator anguli oris, which inserts onto the depressor anguli oris, which actually terminates at the modiolus. In this arrangement, the zygomaticus major has only indirect insertions on the dermis. In contrast, the levator labii superioris alaeque nasi inserts onto the combination of the zygomaticus minor and levator labii superioris, and the whole interconnected complex then inserts into the dermis. Although more detail on the topographic relationships of these direct and indirect cutaneous 
insertions in relation to the NLF would have been helpful, we would interpret these results as suggesting that the levators labii and the zygomaticus minor are primarily responsible for NLF formation, whereas the other muscles have more impact on the position of the oral commissure.

It is premature to draw direct therapeutic conclusions from these findings, yet we believe they can be of use in facial reanimation surgery to better understand the mechanical apparatus responsible for smiling. Likewise, in facial rejuvenation surgery, the surgeon seeking to minimize the nasolabial folds may now be more aware of the groups of muscles responsible for motion in this area, which may be of value in developing more refined neurotoxin, filler, and rhytidectomy techniques. We congratulate the authors on a novel approach and look forward to seeing future studies directed to clinical application of their findings.

Funding No funding was received for this article.

\section{Compliance with Ethical Standards}

Conflict of interest No author has anything to disclose.

Ethical Approval This article does not contain any studies with human participants or animals performed by any of the authors.
Informed Consent For this type of study, informed consent is not required.

\section{References}

1. Gosain AK, Amarante MT, Hyde JS, Yousif NJ (1996) A dynamic analysis of changes in the nasolabial fold using magnetic resonance imaging: implications for facial rejuvenation and facial animation surgery. Plast Reconstr Surg 98(4):622

2. Yousif NJ, Gosain A, Matloub HS, Sanger JR, Madiedo G, Larson DL (1994) The nasolabial fold: an anatomic and histologic reappraisal. Plast Reconstr Surg 93(1):60

3. Yousif NJ, Gosain A, Sanger JR, Larson DL, Matloub HS (1994) The nasolabial fold: a photogrammetric analysis. Plast Reconstr Surg 93(1):70

4. Sandulescu T, Spilker L, Rauscher D, Naumova EA, Arnold WH (2018) Morphological analysis and three-dimensional reconstruction of the SMAS surrounding the nasolabial fold. Ann Anat 217:111

5. Glaser DA, Lambros V, Kolodziejczyk J, Magyar A, Dorries K, Gallagher CJ (2018) Relationship between midface volume deficits and the appearance of tear troughs and nasolabial folds. Dermatol Surg 44(12): 1547

6. Sawh-Martinez R, Lin AM, DeSesa CR, Wu RT, Gary CS, Steinbacher DM (2018) Clockwise and counterclockwise Le Fort I movements influence nasolabial morphology differently. Plast Reconstr Surg 142(6):1572

Publisher's Note Springer Nature remains neutral with regard to jurisdictional claims in published maps and institutional affiliations. 